\title{
Las relaciones de convivencia de los niños, niñas y adolescentes sordos o hipoacústicos: Estudio cualitativo exploratorio
}

\author{
Lourdes Huiracocha T. ${ }^{1}$, Suart Blume ${ }^{2}$, Adriana Orellana P. ${ }^{1}$, Liliana Brito R. ${ }^{1}$, Victoria Abril U. ${ }^{I}$, \\ Elisa Chilet R. ${ }^{3}$, Ruth Díaz G. ${ }^{1}$, Silvia Sempertegui L. ${ }^{1}$, Gicela Palacios S. ${ }^{1}$, Daniela Monsalve $N .{ }^{1}$,' \\ Jorge A. Barahona H. ${ }^{4}$, Sebastián Artega H. ${ }^{5}$, Juan C. Almeida P. ${ }^{6}$, Mirian Huiracocha T. ${ }^{1}$ \\ ${ }^{1}$ Facultad de Ciencias Médicas, Universidad de Cuenca, Av. 12 de Abril s/n, Cuenca, Ecuador. \\ 2 Departamento de Antropología, Universidad de Ámsterdam, Holanda. \\ ${ }^{3}$ Prometeo, Universidad de Cuenca, Cuenca, Ecuador. \\ ${ }^{4}$ Arquitectura Vital, Vicente Cordero 1-55 y Vicente Cuesta, Cuenca, Ecuador. \\ ${ }^{5}$ Carrera de Ingeniería en Marketing, Universidad del Azuay, Av. 24 de Mayo. Cuenca, Ecuador. \\ ${ }^{6}$ Abogados Almeida Pozo, Av. Solano y Remigio Crespo, Cuenca, Ecuador. \\ Autor para correspondencia: lourdes.huiracocha@ucuenca.edu.ec \\ Fecha de recepción: 18 de octubre de 2015 - Fecha de aceptación: 12 de noviembre de 2015
}

\section{RESUMEN}

Estudio cualitativo exploratorio con enfoque fenomenológico que estudió en 10 Niños, Niñas y Adolescentes Sordos / HipoAcústicos (NNA-S/HA) las relaciones de convivencia con la familia, los profesionales de salud, los maestros, los amigos, la comunidad sorda; las cualidades de resiliencia de los NNA-S/HA encontró que las madres aunque tienen altos niveles de estrés son las que afrontan las dificultades y apoyan la intervención, los NNA-S/HA se refugian en sus madres; y los padres se retraen en sus sentimientos y evaden los problemas, y por eso abandonan el hogar al enterarse de que su hijo es sordo. Los profesionales carecen de estrategias para la atención y la comunicación, son distantes del NNA-S/HA, las familias relatan mala calidad en los servicios de salud. Los maestros y los amigos de los NNA-S/HA que van escuelas regulares les excluyen causando convivencias desagradables para los NNA-S/HA. Aquellos que se encuentran en la escuela para sordos se sienten más adaptados, con mejor autoestima y son más resiliente. La mitad de los NNA-S/HA usan dispositivos auditivos pero todos usan o lengua de señas formal o señas familiares para comunicarse con sus amigos y familias. Los NNA-S/HA que usan los dispositivos auditivos no tienen contacto con la comunidad sorda de su región no así los otros que no lo usan y cuya lengua principal es la de señas. Los NNA-S/HA desarrollan como todos los demás sueños, proyectos de vida y luchan por conseguirlo. El estudio permite plantear hipótesis para futuras investigaciones en torno a las estrategias de afrontamiento de las familias, la resiliencia de los NNA-S/HA y la asociación con las relaciones de convivencia.

Palabras claves: Niños, adolescentes, sordera, familia, escuela, servicios de salud, convivencia, lengua.

\footnotetext{
ABSTRACT

Qualitative exploratory study with phenomenological focus probing the relation of 10 Deaf and Hard

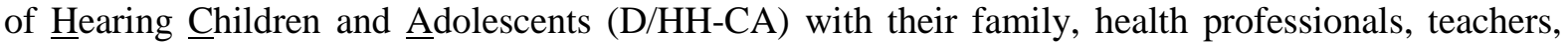
friends and the deaf community; the resilience qualities of those children and adolescents seeking shelter with their mothers who possess relative high levels of stress nurtured by the difficulties to support and intervene with them, while fathers in general retreat in their feelings not seeing the true dimension and leaving home when they learn that their child is deaf. Professionals lack strategies for care and communication, are distant from deaf and hard of hearing children and adolescents, and families are usually disappointed in the poor quality they receive from health services. Teachers and friends of the D/HH-CA avoid them, causing unpleasant feelings and early dropouts from school.
} 
Those who have the opportunity to attend a school for deaf more easily develop resiliency and selfesteem. Half of the surveyed D/HH-CA use hearing aids and all use the sign language or family signs to communicate with family and friends. The D/HH-CA using hearing devices maintain no contact with the deaf community in their region, nor do the children not having access to hearing aids and for whom the sign language is the primary language, maintain contact with the children having hearing aids. D/HH-CA's, like all other children and adolescents, have their dreams and aspirations to make it in life. The survey-based research provided hypotheses to direct in future research on the development of family strategies to cope with deaf and hard of hearing children, to improve the resilience of those children, and to enhance their coexistence and partnership relation.

Keywords: Children, adolescents, deafness, family, school, health services, living, language.

\section{INTRODUCCIÓN}

Los niños, niñas y adolescentes sordos o hipoacústicos no responden a estímulos auditivos porque tienen nula o disminución de la agudeza auditiva (mayor a 30 decibeles en niños) en el oído con mejor audición, lo cual les impide procesar información lingüística, consolidar el aprendizaje de su propio idioma, de enseñanzas básicas, dificultades en la comunicación, generalmente no tienen déficit cognitivo pero podrían tener comorbilidad (autismo, déficit atencional, déficit intelectual u otro) que les ocasione mayor dificultad para el aprendizaje, la realización de las actividades de la vida diaria y la convivencia (Alford et al., 2014). La atención integral temprana que comienza con la comunicación del estado de niño para luego seguir con el diagnóstico, la intervención al NNA-S/HA y a su familia, les ayuda en sus dificultades y en sus capacidades de manera que puedan cursar el mejor potencial de su desarrollo, incluirse en todos los entornos de su vida con independencia y autonomía, tener relaciones cotidianas de convivencia con amigos, personas de la comunidad. El diagnóstico permite conocer si pueden beneficiarse del implante coclear (IC), de audífonos o de otras alternativas y el tipo de lengua que podrán usar para comunicarse (DesGeorges, 2003; Barnett, 2002). En los últimas épocas se promueve el bilingüismo, el oral y el de señas, el oral porque les permite incluirse en los espacios regulares y el de señas porque mantiene la identidad, la cultura de las comunidades de personas sordas y el derecho a la diversidad con las mismas oportunidades en la escuela, en el trabajo, en la comunidad (Fahl et al., 2013; Dominguez, 2009). Los NNA-S/HA como todos los demás aprenden motivados por su impulso interno de socializar, enfrentar retos, buscar lo agradable (juego y arte), solucionar problemas, vivir las relaciones con afecto (emociones, sentimientos y pasiones) lo que les permite ir construyendo un proyecto de vida individual y colectivo como ciudadanos (Huerin et al., 2012; Maddalena et al., 2012).

Parte del desarrollo y la realización de las personas es el vivir con los otros "convivir", a través de las relaciones se recibe y se da afecto, se aprende patrones de entender y resolver la realidad, se contagia del espíritu del valor, de la ética; las relaciones de convivencia permiten ratificar la autoestima, la construcción de los sueños. Las relaciones ideales son aquellas que respetan la diversidad, reconocen las cualidades e impulsan al otro diferente a ser como es y a conseguir su meta; un elemento de las relaciones es la comunicación verbal y no verbal (Kobosko, 2011; Langereis \& Vermeulen, 2015). En el 2013 más del 5\% de la población mundial padeció discapacidad auditiva de los cuales 32 millones fueron niños, la mitad de los casos de pérdida de la audición se pudo evitar mediante la prevención primaria, la mayoría de las personas S/HA viven en países de bajos y medianos ingresos en donde rara vez sus derechos de salud-rehabilitación, educación, participación son respetados (Organización Mundial de la Salud, 2015). En el Ecuador según el CONADIS (Consejo Nacional de Discapacidades) reportó en el 2015 que de un total de 401,538 personas con discapacidad el 13\% tienen discapacidad auditiva de estos el 6.9\% se encuentran en el Azuay (cuarta frecuencia nacional), en el país solo el $0.76 \%$ acude a un sistema educativo y en el Azuay el $7.5 \%$ (CONADIS, 2015), estas circunstancias reales de los NNA-S/HA, de no atención integral de calidad, de un mundo no incluyente sino que les discrimina y priva de todos sus derechos como la educación, les limita el desarrollo terminando con pocas posibilidades de adaptarse a las exigencias del mundo actual (Corvera \& González, 2000). Por esto el NNA-S/HA y su familia suelen tener 
comportamientos de sobreprotección, abandono, aislamiento, el niño vulnerable se aferra con dependencia generalmente a su madre o a otra persona y en sus repetidos fracasos por comunicarse con sus pares tiene desencuentros, desamparo, dificultades en la regulación afectiva y en la vinculación social (Suarez et al., 2006). Un estudio cualitativo realizado en 17 madres e hijos con audición normal comparando con 15 madres y sus hijos con déficit auditivo a través de entrevistas semiestructuras y con observación de la interacción madre hijo durante el juego no encontró diferencias significativas en la expresión de afecto de los niños durante el juego pero si mayor desregulación afectiva en la relación madre niño S/HA porque las madres se quejaron de la conducta de su hijo y los niños respondieron con impulsividad, agresividad debido a la dificultad de comunicarse entre sí (Huerin et al., 2012).

El significado cultural de la sociedad de "discapacitados: enfermitos, inválidos" hace que las familias cuando tienen un hijo con deficiencia auditiva entren en estrés, en conflicto, con temores, mitos que nos les permite seguir el proceso que les lleve a buena calidad de vida (Huiracocha et al., 2013). Las familias quieren que sus hijos S/HA sean lo más pronto "normales" y buscan los audífonos, el IC, las terapias con la intención de que puedan tener lenguaje verbal y evitar la lengua de señas, los padres separan a sus hijos de otros niños S/HA quitándoles la oportunidad de desarrollar la identidad social (Huiracocha et al., 2015). Entonces las familias adoptan con estrés una mezcla de sobreprotección, de exceso de indulgencia pero también las madres incitadas por los educadores pueden convertirse en profesoras de sus hijos con actitudes menos permisivas, más didácticas y menos flexibles (Suarez et al., 2006; Bourke \& Burgman, 2015). El estudio descriptivo longitudinal en 23 madres de hijos oyentes y en 23 madres de hijos S/HA que investigó, a los 22 meses, a los 3 y a los 4 años de edad de los niños, encontró que el estrés de las madres de los niños es permanente y alto en todas las épocas de la vida de los niños, es menor cuando reciben apoyo e intervención; la red social de las madres de niños S/HA es más reducido y generalmente expresan insatisfacción con el soporte social; la mayor causa de estrés es el sentimiento de no poder comunicarse bien con su hijo y la inseguridad de que su niño pueda lograr la independencia y llevar una vida normal (Lederberg \& Golbach, 2002).

Con la edad y el crecimiento los NNA-S/HA deben ir a escuelas inclusivas con maestros y estudiantes que utilicen de señas, con logopedas y con la aplicación de adaptaciones curriculares en aulas habilitadas para ello, cuando esto no ocurre los NNA-S/HA son víctimas de escolaridades tardías o abandonos pedagógicos o son mal catalogados como niños con déficit de atención o con déficit intelectual (Monsalve, 2002). Los NNA-S/HA tienen dificultad para relacionarse con la maestra, con la mayoría de los niños de su edad, falta de aceptación por los demás, de hacer amigos íntimos y de conservarlos, vergüenza de que la gente no los entienda, anhelo de estar con pares S/HA, de que la gente los comprenda e independencia para comunicarse (Ortiz, 2006). Otra de las dificultades más frecuentes es la adquisición de la lecto-escritura debido al reducido repertorio lingüístico-conceptual, el escaso léxico, menor conocimiento de palabras polisemánticas, desconocimiento de expresiones idiomáticas, dificultades con el lenguaje figurativo, formas sintácticas e inferencias (Martinez \& Landa, 2001; Herrera, 2005). La mayoría de NNA-S/HA no presenta problemas en las matemáticas porque no tienen contenidos verbales y más bien les motiva con orgullo a conseguir los logros en el razonamiento lógico matemático (Fuentes, 2000). Tener discapacidad auditiva no quita a los NNA el potencial innato de aprender, de convivir con los demás, de tener sueños, de desear metas y por eso tanto la familia, los profesionales deben estar atentos a las capacidades para fortalecerlas y motivarles a la resiliencia (Domínguez, 2009).

Esta investigación mediante entrevistas semiestructuradas tuvo como objetivos explorar en los NNA-S/HA: (i) las relaciones de convivencia con la familia, los profesionales de salud, los maestros, los amigos, la comunidad sorda; (ii) los NNA-S/HA y sus cualidades de resiliencia.

\section{METODOLOGÍA}

Estudio cualitativo exploratorio con enfoque fenomenológico, que se realizó de octubre 2013 a febrero de 2014 en la ciudad de Cuenca, entrevistando a los padres de 10 NNA-S/HA referidos por 
dos centros educativos, el Centro de Educación Integral de la Universidad del Azuay (CEIAP), centro privado regular que incluye a niños preescolares S/HA y la Unidad Educativa Claudio Neira Garzón (UECNG) escuela especial pública para no oyentes y no videntes, cada centro nos entregó la lista y datos de referencia de los NNA-S/HA, el equipo de investigadores convocó a cada uno de los grupos de padres en el CEIAP y en el UECNG respectivamente, se les explicó sobre la investigación y la importancia para ellos, se tomó los nombres de quienes quisieron participar, se acordaron las fechas de visita a los hogares y antes del inicio de cada entrevista se les hizo firmar el consentimiento informado. Se entrevistaron a los padres de 5 NNA-S/HA que estuvieron en el CEIAP y 5 que estuvieron en UECNG. La condición socioeconómica de los NNA del CEIAP corresponde a clase media, los padres son profesionales y empleados públicos o privados, los padres de los niños del UECNG son de clase baja, los padres tienen empleos informales y el nivel de educación es básico (terminaron la escuela). Los 10 NNA-S/HA estuvieron cursando la escuela, las edades estuvieron entre 6 y 13 años, del CEIAP (se utilizan seudónimos): las niñas Sulata, Tika, Amankaya, Kusisa de 6, 7, 7 y 12 años respectivamente y el niño Kallpa de 10 años de edad; del UECNG: las niñas Tanitani y Utuya de 6 y 13 años respectivamente, los niños Wanka, Waira, Thaluqui de 7, 11 y 13 años de edad. Los NNA-S/HA del CEIAP todos estuvieron recibiendo terapias y tenían aparatos tecnológicos (IC o audífonos), para escuchar-hablar y no tuvieron contacto con la comunidad de personas sordas de la región, mientras que los de la UECNG aunque algunos tienen los aditamentos todos usan lengua de señas y tienen contacto con la comunidad de personas S/HA. En dos de las 10 entrevistas participaron los padres y madres, en una entrevista solo el padre, en una los dos padres y un abuelo, en una la madre sorda con los dos abuelos maternos oyentes, y en 5 restantes solo las madres. Las cinco familias procedentes del UECNG recibían por ser pobres el bono económico del gobierno denominado "Bono de desarrollo humano" y ninguna de las familias del CEIAP lo recibió. La mamá y el papá de Tika son S/HA como ella, los demás padres y madres son oyentes. La identificación del déficit auditivo fue en nueve casos realizados por la mamá y en Tika por los abuelos, el diagnóstico se hizo en los cinco casos del CEIAP alrededor de los 7, 8 meses de edad y en los cinco de la UECNG desde el 1 hasta los 3 años de edad. Amankaya tiene también síndrome de Ret (autismo y déficit intelectual) y Wanka tiene parálisis cerebral y déficit intelectual, Thaluki ve solo con un ojo. El equipo de investigadores estuvo conformado por una pediatra, un antropólogo, un abogado, dos educadoras especiales, una psicóloga, dos intérpretes certificadas en lengua de señas (una educadora especial y la otra socióloga), un arquitecto, un estudiante de marketing que en la ejecución de la investigación aprendieron la importancia de que sean inclusivos los espacios físicos y las etiquetas de todo el ambiente. Las profesionales intérpretes fueron quienes hicieron las entrevistas en un solo tiempo (entre 18 y 60 minutos) que fueron grabadas y transcriptas. El equipo de manera conjunta hizo manualmente la codificación y el análisis de los datos, se triangulo y se consultó con expertos.

\section{RESULTADOS Y DISCUSIÓN}

\subsection{La familia}

"De que yo me acuerdo nadie me dijo -te vamos a apoyar- yo siempre sola con las cosas de mi hijo, hasta ahora nunca se involucran en el lenguaje de él” (Madre de Thaluqui).

Seis de los diez NNA-S/HA conviven en hogares nucleares, uno tiene un hogar expandido (madre, padre y abuelos), tres viven solo con la mamá porque fueron abandonadas por sus esposos luego de enterarse que el NNA es S/HA, solo dos padres se involucran en el cuidado de sus hijos S/HA, los demás se mantienen distantes sin apoyar a la madre. En todos los casos la mejor forma de comunicarse con la madre (la persona más cercana a ellos) es el lengua de señas, seis NNA reciben apoyo de sus hermanas, los hermanos son distantes. Los familiares como tíos, primos y otros tienen pena de los NNA-S/HA y algunos niños pequeños de la familia se ríen de ellos, dos hogares se sienten apoyados por tías cercanas, y en tres casos la madre siente que sus familiares los excluyen porque tienen un NNA-S/HA. 
"por ejemplo las tías en el bus ya le ven, se esconden, se van, yo les he visto, tienen vergüenza, ya no es lo mismo, solo dicen -hola- pero no es como con los otros sobrinos" (Padre de Tanitani).

"Mamá yo quiero que mis primos jueguen conmigo, quiero estar con ellos..., mi hijo me dice con señas...” (Mamá de Kallpa).

El padre de Tika también S/HA, no aporta económicamente, los abuelos oyentes educan a Tika y desplazan en las decisiones a la madre sorda quien se revela pero sin tener éxito por esto no tiene cercanía con su hija, Tika convive entre el conflicto de las expectativas de su madre (recate de la identidad de personas S/HA) y sus abuelos (camino hacia la normalidad). Las nueve madres de los NNA son afectivas con sus hijos o hijas, son las primeras en sospechar el déficit auditivo, llevan el proceso diagnóstico y de intervención, aprenden la lengua de señas, defienden a sus hijos e hijas con todas las fuerzas, los NNA-S/HA se refugian en sus madres cuando sufren. Las madres que reciben apoyo de sus familiares se sienten más seguras en las decisiones con sus hijos S/HA. La mayoría de las madres de los NNA-S/HA evitan que vayan solos a espacios nuevos porque tienen miedo de que les pase algo pero cuando van a la escuela les motivan a la integración, la madre de Waira retrasó su ingreso al sistema escolar por miedo al maltrato. Las madres sacrifican su tiempo libre, sus sueños personales por su hijo, son quien les llevan a las terapias, a la escuela, quienes afrontan los problemas y en muchas ocasiones hacen de profesoras, motivan a sus hijos a avanzar y festejan los pequeños logros. En dos casos los padres aportan con el cuidado. La mamá de Sulata:

“...prácticamente pase sola en la búsqueda, porque como mi esposo trabajaba, prácticamente yo estuve sola... él le dice a uno -que no, que usted es demasiado preocupada- o que exagero... yo si quisiera organizar un viaje no puedo, porque tengo que siempre estar con ellas, con mis hijas, la familia" (Madre de Sulata).

Los padres de Amankaya y las madres de Wanka y Thaluki (niños también con otro déficit) observan las potencialidades de sus hijos y tienen la misma esperanza que los otros padres: "independencia" y "vida digna".

“...Que él sea un abogado, o un arquitecto, eso es lo que yo espero y aspiro para él, por eso le apoyo mucho...” (Padre de Wanka).

Los miembros de las familias reaccionan frente al nacimiento de un NNA-S/HA de acuerdo a: (i) las estrategias de adaptación (schock-dolor, reconocimiento de la condición del hijo, negación, acción constructiva de toma de decisiones para la intervención) (Fadda, 2011), (ii) a la personalidad (neurótica, extrovertida, responsable, abierta al cambio, amable) (Plotkin et al., 2014) y (iii) a los estilos de afrontamiento ("centrados en sus propios sentimientos y emociones", "evasión de los problemas "esfuerzo por buscar soluciones a los problemas) (Kobosko, 2011). Para apoyar óptimamente a las familias en el año 2012 en Australia se reunieron equipos internacionales de expertos conformados por padres, profesionales S/HA, líderes y especialistas en intervención temprana e investigadores con experticia en trabajar con personas S/HA, familias, comunidades de personas sordas y elaboraron las guías de buenas prácticas para las familias considerando la evidencia para conseguir: (i) roles parentales con una comunicación placentera con los hijos $\mathrm{S} / \mathrm{HA}$; (ii) el buen vivir de las familias: disfrutando a los niños S/HA, estableciendo relaciones estables, teniendo la disponibilidad emocional para ver con optimismo el futuro del niño S/HA; (iii) compromiso: activa participación en programas, elección de la información, decisiones, protección del niño; y (iv) autoeficacia: para que los padres promuevan el desarrollo del niño S/HA. Para lograrlo los profesionales cercanos a las familias deben considerar los valores, la cultura de las familias, los objetivos y aspiraciones de vida (Moeller et al., 2013). En ninguna de las familias de esta investigación se aplicaron estos criterios por eso los NNA-S/HA permanecen replegados en la madre 
y alejados de la convivencia familiar, los miembros tienen conflictos en asumir nuevos roles y estrategias de adaptación y aunque todos tienen alto estrés, los padres y hermanos permanecen en el estado de negación, centrados en sus propios sentimientos y evadiendo los problemas por eso abandonan el hogar o se mantienen alejados, en cambio las madres y las hermanas con personalidades responsables tienen acciones más constructivas al asumir la intervención y ven los logros del NNA S/HA, enfrentan los problemas. La investigación en Polonia indica que todos reaccionan con estrés pero que las mujeres adoptan más el estilo de centrarse en sus sentimientos o evadir los problemas de manera más frecuente que los hombres (Kobosko, 2011), mientras que Suarez en su investigación cuanti cualitativa reporta que las madres son las que afrontan el cuidado de sus hijos S/HA.

El estudio cuantitativo que investigó la personalidad y el estrés en 114 padres y la conducta de sus hijos S/HA de 3 a 10 años de edad reportó que el más alto nivel de estrés estuvo en los padres neuróticos, demostrado en la ejecución de cada una de las actividades de la vida diaria (relaciones familiares y maritales, finanzas, disciplina, rutinas, educación, excursiones) y aunque mostraron responsabilidad con sus hijos S/HA el estrés aumentaba frente al uso de dispositivos auditivos, IC, educación y lengua de señas. El estrés de los padres producía menor eficiencia en su rol de cuidado y menor visibilidad de los logros de su hijo y en los niños producía reactividad, introversión o problemas de conducta. Mientras que personalidades parentales extrovertidas y abiertas al cambio con menos estrés generaban mayor solvencia no solo en el cuidado del niño S/HA sino también en las actividades de la vida diaria (Plotkin et al., 2014).

\title{
3.2. Los profesionales de salud
}

En ninguno de los casos el equipo de salud detecta las dificultades de audición del NNA-S/HA, casi siempre es la madre en un caso el padre y en otro los abuelos quienes observan que el niño o la niña no tiene un desarrollo normal, momento en el cual empieza un trajinar por los servicios de salud que no son asertivos ni les apoya, la información que reciben sobre la discapacidad auditiva es por amigos. Los médicos no tienen ningún acercamiento con el NNA-S/HA, no les evalúan integralmente. El papá de Tanitani expresa lo siguiente:

\begin{abstract}
“...Otra vez prendo el radio a todo volumen, mi otra guagua se asusta, llora y mi otra hija no se reviraba para nada, le digo a mi esposa - creo que ella padece del oído, no oye, no hablaba nada-, luego yo le lleve al Centro de Salud a Lazareto y les digo a los doctores: -señorita, creo que mi hija no escucha, es sorda-, me hicieron unos exámenes allí, pero los médicos me dijeron: -no, ella es normal, algunas niñas hablan hasta 4 o 5 años, ella no quiere hablar no más, es majadera-...
\end{abstract}

Las guías de buenas prácticas permiten la temprana detección, diagnóstico e intervención del NNA-S/HA, apoyando en la diversidad cultural y económica a las familias a tomar las mejores decisiones, el entendimiento y el cumplimiento de compromisos (Moeller et al., 2013). Así lo demuestra el estudio cualitativo sobre las percepciones que tienen los padres sobre la atención quienes relatan malas experiencias en el trato y en la comunicación (dificultad de leer los labios de los médicos ya sea por el uso de mascarillas o porque no hablan de frente o lo hacen rápido, mal uso de la lengua de señas) y hacen recomendaciones que están consideradas en las guías (DesGeorges, 2003).

En Estados Unidos a pesar de que la pérdida auditiva es la sexta condición crónica más común, los médicos aprenden el proceso de diagnóstico pero no tienen en su currículo de formación el acompañamiento psicológico y la comunicación con personas S/HA (lectura de labios, lengua de señas) causando frustración o alejamiento entre el médico, la persona sorda y su familia. Fortalecer las destrezas de comunicación en el personal de salud incluyendo en la docencia a personas S/HA que empleen lengua de señas o que el estudiante en una de sus rotaciones pueda participar en las comunidades sordas para entender la cultura del grupo (Barnett, 2002). Una investigación cualitativa indica que las personas sordas que usan lengua de señas han tenido las mejores experiencias cuando durante la atención existen intérpretes certificados que facilitan la comunicación (Steinberg et al., 2006). Ninguna de las familias de esta investigación tuvo el apoyo de personal médico que conocía la 
cultura o el lenguaje de personas S/HA, el personal médico no establece una cercanía de convivencia con los NNA-S/HA.

"No...no, no le toca, ni le mira, ni le examina....mi hija hace como si el doctor no existiera..." (Madre de Tika).

\subsection{La escuela, los maestros, los compañeros, los amigos}

Los NNA-S/HA que fueron al CEIAP en la edad preescolar recibieron el apoyo terapéutico y las adaptaciones curriculares por parte del centro y luego se trasladaron a otro centro educativo regular para cursar la etapa escolar. Los NNA-S/HA de la UECNG antes de ingresar al centro tuvieron experiencias previas negativas de paso por escuelas regulares. Todas las familias expresan que en las escuelas regulares los maestros no están preparados ni se esfuerzan porque no elaboran adaptaciones curriculares, no tienen letreros en braille, no utilizan lengua de señas ni conocen el manejo de audífonos o de implantes cocleares y más bien excluyen a los NNA-S/HA de las actividades grupales o deportivas por miedo a que estos implementos se dañen. Todas las familias esperan que las escuelas ayuden a los NNA-S/HA a ser independientes, fortalezcan la autoestima y les de las oportunidades de cumplir los sueños de los NNA-S/HA pero se preocupan por el futuro educativo porque no existe un colegio o universidad que les dé cabida, El papá de Amankaya, niña también con trastorno motriz y déficit intelectual, dice:

“... por ejemplo le mandan un deber a que se aprenda un poema, es una niña que no va a poder, probablemente nunca, entonces lo que quiero decir no hay inclusión..." más adelante indica: “... .me contó un padre que el profesor de su hijo S/HA hablaba de espaldas y el niño S/HA trataba de verle los labios para ver si puede entender, en cambio el profe se da la vuelta y le pregunta algo al niño y el niño no puede y le trata mal, y dice si oyes, si oyes, ya no me molestes..." (Padre de Amankaya).

El apoyo de los padres, el trabajo en la diversidad ayuda a los NNA-S/HA a aceptar su condición:

“...Mamá yo quiero nuevos amigos... dice Kallpa... cuando salimos al parque se hace amigo de alguien y les dice "yo no escucho", les explica, no le molesta ni se siente mal..." (Padre de Kallpa).

Los NNA regulares realizan bullying a los NNA-S/HA, no les incluyen en el juego, en las invitaciones a casa, Las hermanas son las defensoras del maltrato en los casos en los que comparten la escuela:

“...mamá yo quiero que los niños no me vean como enferma... me cuenta mi hija... y yo he visto como los compañeros dicen: -no te mates hablando con ella, porque ella no te escucha, no le digas nada, déjale no más sentada, ella es sorda muda, ella es enfermami hija como que ella ya veía los labios y entiende que palabra quería decir, y ella se siente mal, ya ve la reacción de la persona, mejor se iba a sentar era cohibida..." (Madre de Utuya).

La UECNG ofrece una educación dirigida a las necesidades de los NNA-S/HA, tienen talleres para enseñar a las familias la lengua de señas; los entrevistados no se quejan de este centro educativo más bien expresan que sus hijos se sienten tranquilos y adaptados. Los NNA-S/HA y sus padres que vivieron las experiencias en sistemas regulares logran estar más tranquilos porque en la UECNG ya no son discriminados. Las relaciones de amistad no son conflictivas, los NNA-S/HA tienen mejores lazos de amistad con sus pares, ninguno de ellos expresa bullying, los adolescentes tienen relaciones de enamoramiento, momentos recreativos entre ellos, sin sufrir frustraciones y cuentan experiencias 
parecidas a las de los adolescentes no S/HA. Los NNA-S/HA pueden tener mejores relaciones con adultos y niños no S/HA en otros espacios porque se sienten diferentes pero no inferiores, su autoestima les permite quererse como son:

\begin{abstract}
"Mamá yo quiero tener muchos amigos, me pide Thulaqui... Sí, él es muy amiguero, se lleva con todas las edades, tiene amigos de la edad de él, con adultos, así. Él les hace señas y los otros ya dicen que le entienden..." (Madre de Thaluqui).
\end{abstract}

A todos los NNA-S/HA les gusta ir a su escuela, las mayores dificultades que tienen son las que se relacionan con la lectoescritura, algunos son buenos en matemáticas. El círculo de amigos de los NNA-S/HA que no es muy amplio, las mejores amigas son las hermanas, luego algunos primos, amigos de un grupo religioso y en los que se encuentran en la UECNG sus compañeros, el que los NNA no S/HA no conozcan la lengua de señas se convierte en una dificultad para la comunicación, el juego; la mayoría de NNA regulares piensan que la sordera es una enfermedad y esto les causa miedo, muy pocos NNA regulares tienen gestos solidarios. La guía de buenas prácticas promueve en los educadores competencias teóricas y prácticas actualizadas que trabajando con un equipo interdisciplinar profesional optimiza el potencial de desarrollo de los NNA-S/HA y sus familias así como el desarrollo de habilidades de relación de los NNA oyentes con los NNA-S/HA. Lo óptimo de una educación inclusiva es que se revise continuamente la cultura escolar en busca de las barreras, promoviendo actitudes positivas hacia la diversidad, desarrollando sistemas de comunicación compartidos (oral, de señas o mixto), con herramientas y mecanismos para optimizar el lenguaje y la comunicación tales como dispositivos auditivos, implantes cocleares, sistemas de frecuencia modulada, tecnología visual (textos, alertas visuales, videos) (Moeller et al., 2013) para establecer interacciones con su entorno social, con personas oyentes y sordas, preparando los contenidos curriculares para el aprendizaje de la lengua oral y escrita. Ofrecer a los alumnos una imagen en positivo, un sentimiento de pertenencia para favorecer la cooperación, la autonomía y la asunción de responsabilidades (Domínguez., 2009). Sin embargo los NNA-S/HA de esta investigación no se sienten incluidos en las escuelas regulares porque no se cumplen estas buenas prácticas y los NNAS/HA tienen convivencias desagradables en sus entornos escolares.

En Estados Unidos el 40\% de los NNA-S/HA están en escuelas regulares y en el Reino Unido el $85 \%$ en contacto con NNA oyentes, varios estudios indican que los NNA-S/HA son más rechazados que sus pares oyentes (Martin \& Bat-Chava, 2003). Los NNA-S/HA con IC o dispositivos auditivos hacen el esfuerzo por ser incluidos y ser considerados como los demás para tener la probabilidad de ser populares como los niños oyentes. El estudio cuantitativo realizado en 58 niños S/HA prelinguales con IC unilateral colocado antes de los 30 meses de edad y con inteligencia no verbal normal, sin ningún otro desorden como autismo o déficit de atención, de tres ambientes: (i) escuela regular, (ii) escuelas para niños HA que usan lengua de señas y lenguaje oral, (iii) escuelas para niños sordos solo con lengua bilingual de señas (Holandes y Alemán) encontró que los niños del primer grupo fueron más resilientes que los del tercer grupo porque existió asociación entre las habilidades de percepción del lenguaje oral y el buen vivir de los niños, pobre comunicación y cualidades de lenguaje con más problemas de conducta. Sin embargo para NNA-S/HA la escuela de sordos les ofrece un ambiente que les protege y les educa acorde a sus necesidades (Langereis \& Vermeulen, 2015). Los NNA-S/HA de nuestro estudio que están en la escuela para personas S/HA son más resilientes.

\title{
3.4. La comunidad sorda y la lengua de señas
}

Los NNA-S/HA que pertenecen a la clase media, que tienen audífonos o IC, reciben terapias para hablar, de manera oficial no utilizan lengua de señas (lo usan en su cotidianidad como una herramienta fácil y agradable de comunicación) por lo tanto ninguna de las familias hace el esfuerzo por aprender la lengua de señas. Las familias de estos NNA no les llaman "S/HA" sino NNA con "discapacidad auditiva" porque consideran que "S/HA" es una palabra peyorativa que no les da esperanza. Ni los NNA-S/HA incluidos en sistemas regulares ni las familias tienen contacto con otras personas S/HA, esto les hace sentir solos, rechazados pero pueden visibilizar que viven en una sociedad no incluyente; algunos de los padres y madres, los de mejor nivel educativo y condición 
socioeconómica, luchan por una sociedad más diversa, los derechos de sus hijos y por más oportunidades, tal es el caso de los padres de Kallpa que luego de que los niños salieron del CEIAP y fueron a diferentes escuelas regulares, para que sus hijos S/HA se mantengan en contacto, organizaron un paseo pero ninguna de las familias asistió; también se han enterado que han realizado fiestas infantiles (cumpleaños) y no invitan a NNA-S/HA (entre ellos a Kallpa). La familia de Amankaya formó una Fundación denominada "Escúchame" que va creciendo:
“...la Michi se ha movido por todos lados, ha formado la fundación, la Michi es la presidenta, estamos contentos, es decir ha habido apoyo, creemos que cada vez el Ecuador va cambiando y creemos que hay más apertura para la gente con discapacidad, no solo la sordera." (Padre de Amankaya).
“...es por esa razón que incluso cuando pasamos todo este proceso mucha gente igual que nosotros queríamos crear una fundación para dar curso a este soporte psicológico, emocional y espiritual..." (Padre de Kallpa).

Los NNA-S/HA que acuden a la UECNG tienen contacto con la comunidad sorda, las familias en las reuniones comparten sus temores, expectativas, sueños, esto les causa un sentimiento de solidaridad entre ellos aunque se diluye en la práctica por las ocupaciones laborales de subsistencia de la madre. Las familias y NNA-S/HA se sienten menos solos y menos raros. La lengua oficial es la de señas, no existe problema en la aceptación de este tipo de comunicación y no compiten por ser "normales". La madre y las hermanas son las que aprenden la lengua de señas mientras que la mayoría de padres no hacen el intento o piensan que a su edad es muy complicado. Los problemas de estas familias se relacionan más por su condición de pobreza y no poder pagar servicios de salud, educación y otros. El conflicto entre el padre y la madre S/HA de Tika y sus abuelos oyentes es que mientras los padres piensan que Tika estará mejor en contacto con la comunidad sorda y usando la lengua de señas, los abuelos quieren "normalizarla" en un mundo solo de oyentes.

Existen algunas corrientes en la intervención de los NNA-S/HA, entre ellas, la audiológica que define a la sordera como una discapacidad que ha de ser tratada con implementos tecnológicos y con rehabilitación para la recuperación de las destrezas auditivas verbales para la completa integración social, y otra corriente la sociocultural que define a las personas sordas no por lo que les falta (la audición), ni por lo que no son (oyentes), sino por lo que son, personas con capacidades que además comparten con otros semejantes una lengua, una historia y una cultura propia, que les confiere una "identidad" que debe ser aceptada y reconocida en una sociedad que abogue por la "igualdad en la diversidad". Las personas sordas Los NNA-S/HA deberían ser educadas desde las dos perspectivas adoptando un concepto multidimensional en el que los NNA-S/HA tienen dificultades pero también capacidades para lograr un desarrollo óptimo y una comunicación que les permita tener una vida digna. Por eso las personas sordas se definen como bilingües y biculturales, en el sentido de que deben aprender las dos lenguas de señas y oral y que pueden sentirse activos en comunidades de oyentes y en la comunidad sorda (Fadda, 2011).

En las sociedades actuales, el acceso a la información y a las comunidades es predominantemente auditivo por lo que resulta muy limitado o inexistente para muchas personas sordas que desconocen incluso lo que sucede en la comunidad inmediata y más aún en el resto del mundo, esta barrera de comunicación es lo que pone más barreras a las personas sordas e incrementa el riesgo de ser discriminadas y excluidas socialmente. En el mundo se estima que existen 70 millones de personas sordas que usan lengua de señas pero no se sabe cuántas comunidades sordas existen, lo que se convierte en una dificultad para la planificación. La World Federation of the Daef (WFD) en el 2009 publicó un estudio sobre la situación actual de los derechos de las personas sordas en el mundo, en 44 de 93 países hay un reconocimiento legal de la lengua de señas y solo 47 poseen legislación en materia laboral que proteja a las personas usuarias de estas lenguas, en 31 países a las personas sordas no se les permite obtener el carnet para conducir y tan solo 18 cuentan con un servicio de interpretación de lengua de señas, con formación profesional y un código ético propio, solo en 23 países las escuelas ofrecen una educación bilingüe (lengua de señas y oral) y por eso hay un gran porcentaje de analfabetismo en este grupo (Muñoz-Baell \& Ruiz-Cantero, 2011). 


\subsection{Los NNA-S/HA y sus cualidades de resiliencia}

Los NNA-S/HA desarrollan sus habilidades como todos los demás a través del juego, del afecto, de la curiosidad, de las travesuras y de la necesidad de resolver problemas, buscan relacionarse con su familia, con los NNA de su edad, hacen esfuerzos por comunicarse.

"Papá yo quiero... me dice Tanitani... pero yo no le entiendo siempre... pero ella me muestra el celular y ya me enseña lo que quiere... yo ya le entiendo..." (Padre de Tanitani).

La forma más común de comunicarse es por la lengua de señas, incluso aquellos que tienen audífonos o implantes. En la escuela frente a los problemas en la lecto escritura eligen asignaturas que pueden como las matemáticas, los deportes, el arte, la computación, se esfuerzan por aprender. Luchan contra los temores de sus padres para ser independientes y darles alegrías con sus logros:

“...mamá yo quiero cuidarte cuando seas viejita... me dice Waira ... yo llego a la casa solo para calentar la comida para darles de comer y él ya ha dado de comer a mis pollos, a mis cuyes, al chancho todo yo le digo no te vayas a trabajar y me dice no mamá yo trabajo" (Madre de Waira).

Sienten que ser S/HA no es una limitación para aprender pero se frustran y disminuyen sus esfuerzos cuando los otros les identifican como personas que no escuchan, no hablan, les aíslan por ser diferentes; algunos lloran, otros se aíslan y pocos expresan rabia cuando no pueden algo y no reciben ayuda, esto les ocasiona depresión, disminución de la autoestima y temor para lo nuevo pero terminan positivamente adaptándose cuando el ambiente cambia de agresivo a acogedor.

“...Sufrió bastante, no se adaptó pronto, lloraba, no se quería quedar, no le gustaba, peleaba y antes mismo de entrar a la escuela, era bien agresivo, porque no se comunicaba y el reaccionaba. Luego en la escuela poco a poco fue aprendiendo las señas, yo también fui aprendiendo, ya cuando nos comunicábamos ya fue tranquilizándose, entonces si le toco adaptarse a la escuelita..." (Madre de Thaluqui).

La mayoría de los NNA-S/HA adoran a sus madres, con ellas se sienten seguros, protegidos y son cariñosos con todos aquellos que les permiten acercarse entregando abrazos. Lo que más les hace felices es cuando otros NNA les incluyen en las actividades recreativas y de participación grupal. Todos los NNA-S/HA tienen buena conducta, ninguno de ellos es conflictivo más bien en ambientes nuevos son tímidos y miedosos. Se esfuerzan por conseguir logros y metas en la escuela, en la casa.

“...mamá yo quiero la compu... dice Sulata... Ah no, le encanta, o sea ella solita, entra en el internet, pone las palabras del juego que a ella le gusta, pone un juego de vestirles a unas niñas, entonces juega un rato que no pasa de los 20 minutos, entonces ella mismo sale del juego, apaga la computadora,... ella sabe muy bien, sabe las letras, los números, escribe todo, está muy bien..." (Madre de Sulata).

Las madres de las adolescentes Utuya de 13 años y de Kusisa de 12, que usan lengua de señas y están en la UECNG, han dejado de ser niñas y como todas las adolescentes se apartan de sus madres, buscan amigas de su edad, les gusta compañeros, sus madres tienen temores, les apartan del círculo de amigos, les prohíben el Facebook, Utuya reclama su espacio y confianza, solicita que su madre no le subestime. 
“...mamá yo quiero conversar con mis amigos... dice Utuya... yo a veces si le suelto un poco, pero como es mujercita siempre le tengo cuidado le digo que no tiene que estar ahí conversando mucho con los chicos, que me avise si le hacen algo... ella se lleva con los chicos, a lo menos el Edwin que ya tiene 18 años... yo encontré unas cartas escritas que tuvo en la mochila de ella, yo realmente nunca le revisaba, pero un amigo me dice que le revise los cuadernos, yo dije no porque yo confió en mi hija, que no, que tenga cuidado porque a veces los hijos a uno le miente, entonces empecé a revisar encontré esas cartas y vine a hablar con la profesora, lo que pasa es que ella esta ilusionada... entonces de ahí Utuya me dijo que no le subestime, ...y a me di cuenta que mi hija tiene una inteligencia barbará...". (Madre de Utuya).

“...nos pusimos a conversar las mamas, y las mamitas decian por alli no nuestras guaguas son bien enamoradizas, y yo decía chuta mierda lo que me espera, ella esta chiquita todavía decía y ahora Dios mío cuando crecerá, y ahora yo que hago, bueno y ahora ella ya está creciendo y yo, yo a ella le veo en la computadora en el Facebook y yo le digo que estás haciendo?, ¿con quién estas conversando?, ¿Quién es?, hay, mami no molestes, estoy mirando..." (Madre de Kusisa).

Mientras que las madres de Thaluki de 13 años y de Kallpa de 10 años están contentas que sus hijos estén enamorados y les dan consejos para que su comportamiento sea adecuado:

“...Mamá yo quiero a mi novia... dice Thaluki... Sí, yo digo que sí, si el ahora ya tiene novia, él está enamorado de una compañera y como un buen muchacho tiene una sola novia..." (Madre de Thaluki).

Aunque no todas las familias tienen la oportunidad de poseer, a todos los NNA-S/HA les gustan los aparatos electrónicos como radio, televisión, computadora, celulares, la mayoría maneja bien el internet (si no hay una en casa van a los sitios donde pueden usar computadoras e internet). A la mayoría de los NNA-S/HA les gusta lucir bien, limpios, con linda ropa. A todas las familias de los NNA-S/HA les preocupa la adolescencia, el ingreso al colegio y universidad, el enamoramiento, el matrimonio, el futuro de ellos porque la sociedad no es inclusiva; la mayoría de los NNA-S/HA sueña con una profesión y hablan con sus madres de esa meta.

“...mami yo cuando me casé, me voy a vivir en la playa y voy a preguntarle a mi esposa si quiere... es que cuando tenga hijos. Voy a ser chef...” (Kallpa).

Se narran dos tipos de afrontamiento: (i) el afrontamiento del problema con exteriorización (planificación, conducta asertiva frente al estresor), y (ii) el afrontamiento con interiorización de las emociones (regular la emoción frente al estresor) encontrándose mayor éxito en el primer tipo de afrontamiento. Los adolescentes utilizan las siguientes estrategias: (1) recursos personales como familia, para resolver los problemas, (2) diversión y las respuestas emocionales para disminuir el impacto de los estresores, (3) la combinación de las dos anteriores Los NNA-S/HA se sienten más cómodos cuando están en contacto con sus pares S/HA y tienen más éxitos en escuelas regulares. Las mujeres son más confidentes, sumisas, utilizan más estrategias emocionales y de cooperación, hacen grupos más pequeños y los niños son expresivos, hacen grupos más grandes como los equipos deportivos porque en el juego se usa el lenguaje corporal (Martin \& Bat-Chava, 2003).

Los adolescentes S/HA con el inicio de la pubertad deben encaran patrones más complejos de interacción social en todos los entornos de convivencia; la imagen corporal, la autoestima son ingredientes indispensables para el fortalecimiento de valores, de la ética personal, de decisiones en el desarrollo de relaciones más intensas con jóvenes, el coqueteo, el noviazgo, el encuentro en grupos juveniles. El adolescente S/HA puede sentirse discriminado, deprimido cuando sus pares no respetan la diversidad ni hacen el esfuerzo por la inclusión (Corvera \& González, 2000). El estudio cualitativo 
fenomenológico que exploró las experiencias de bullying de los NNA con discapacidad (entre ellos auditiva) en el colegio mediante entrevistas a 10 niños de 8 a 10 años de edad reporta que todos tuvieron experiencias de bullying de tipo verbal, física y psicológica debido a su aspecto físico o al uso de aditamentos de apoyo, los pares rumoran por detrás de los NNA-S/HA, no quieren compartir con ellos, cuestionan el apoyo adicional que reciben en clases o en terapias, los acosos psicológicos no son detectados por el profesor debido a la falta de manejo con efectividad de la clase y de la discapacidad; estas situaciones que les lastima origina en los niños enojo, algunos reaccionan con violencia, otros ignoran el maltrato, otros se refugian en sus mascotas, otros hablan con quién les da soporte. El estudio explica la importancia de los amigos, los NNA-S/HA prefieren a aquellos que no se burlan de ellos, que les apoyan, que les cuidan; los NNA-S/HA sienten que un buen amigo les ayuda en su autoestima y en la consecución de sus metas. A su vez los niños con discapacidad son solidarios con otros niños que tienen problemas. Solo uno de los padres de los 10 niños fue a la escuela a conversar sobre el bullying a su hijo (Bourke \& Burgman, 2015). Los NNA-S/HA de esta investigación son resilientes y presentan diferentes estrategias de afrontamiento, ellos desarrollan mecanismos para hacer que su convivencia sea agradable e incluyente.

\section{CONCLUSIONES}

Los NNA-S/HA y las familias de este estudio, sobre todo las madres, aplican estrategias de afrontamiento de la discapacidad auditiva. Las escuelas regulares y los profesionales de salud no aplican las guías de buenas prácticas de la atención por esto los NNA-S/HA no tienen las mismas oportunidades que tienen los otros NNA en salud, educación, participación, ocio y otros. Los NNAS/HA tienen una convivencia cercana a las personas que les prestan afecto y solidaridad, adquiriendo habilidades sociales y de comunicación que les permite incluirse, aprender y obtener independencia en sus actividades de la vida diaria, sueños y proyectos de vida. Los testimonios hablan sobre la resiliencia de los NNA-S/HA y de sus madres. La lengua de señas y las señas familiares siguen siendo la principal forma de comunicación de los NNA-S/HA a pesar que la mitad de ellos utilizan dispositivos auditivos. Los NNA-S/HA se adaptan mejor cuando sus amigos les incluyen y cuando comparten con pares S/HA.

Para mejorar la calidad de vida eliminando la exclusión, discriminación y fomentando el óptimo desarrollo se recomienda seguir los diez principios de "Buenas Prácticas para los NNA-S/HA y sus familias" que son: (i) atención temprana (diagnóstico, intervención) y acceso equitativo a los servicios de salud; (ii) proveer soporte emocional a las familias para favorecer la interacción con el NNAS/HA, para que identifiquen necesidades, prioridades, deseos, esperanza; desarrollen estrategias de adaptación, afrontamiento, resolución de conflictos; (iii) entregar a las familias la suficiente información para la toma de decisiones, incluyendo educación sobre leyes, derechos para el buen vivir de los NNA-S/HA; (iv) incorporar a las familias a un sistema de apoyo que les permita ganar conocimientos y experiencias para que ellos y sus hijos S/HA actúen con independencia y autonomía; (v) proveer maestros, ambiente y herramientas para el aprendizaje del bilingüismo (oral y de señas), bicultural (comunidad sorda y de oyentes); (vi) uso de la tecnología para favorecer la comunicación: audífono o implante coclear $\mathrm{u}$ otros dispositivos visuales o auditivos y el aprendizaje computadoras o teléfonos; (vii) optimizar el desarrollo de los NNA-S/HA: entrenando, al personal de salud, a los educadores para una óptima interrelación que favorezca la intervención; (viii) impulsar el trabajo colaborativo de los profesionales para favorecer el proceso de desarrollo y de aprendizaje del NNAS/HA en la escuela, en la familia y en otros ambientes; (ix) evaluar continuamente los logros y dificultades de los NNA-S/HA y de sus familias para intervenir oportunamente; y (x) realizar seguimiento a los programas de atención a los NNA-S/HA en la búsqueda de correctivos.

Aunque estos resultados corresponden a un estudio exploratorio en 10 casos y que pueden entenderse solo en contextos y en situaciones similares a las descritas sin embargo los datos orientan al desarrollo de investigaciones cualitativas más complejas (interpretativas, predictivas) en una población mayor y en un período más prolongado para la comprobación de hipótesis sobre las 
estrategias de afrontamiento de las familias, la resiliencia de los NNA-S/HA y su asociación con las relaciones de convivencia de los NNA-S/HA.

\section{AGRADECIMIENTOS}

Los autores agradecen al CEIAP y a la UECNG, a la Asociación de Personas Sordas del Azuay (APSA) a los NNA-S/HA y a sus familias que permitieron testimoniar sus experiencias de vida.

\section{BIBLIOGRAFIA}

Alford, R., K. Arnos, M.W. Fox, J. Lin, C. Palmer, A. Pandya, H. Rehm, N. Robin, D. Scott, C. Yoshinaga-Itano, 2014. American College of Medical Genetics and Genomics: Guideline for the clinical evaluation and etiologic diagnosis of hearing loss. Genet. Med., 16(4), 347-55.

Barnett, S., 2002. Communication with deaf and hard hearing people: A guide for medical education. Academic Medicine, 72(7), 694-700.

Bourke, S., I. Burgman, 2010. Coping with bullying in Australian schools: how children with disabilities experience support from friends, parents and teachers. Disability and Society, 25(3), 359-371.

CONADIS, 2015. Consejodediscapacidades.gob.ec [página en Internet]. Estadística personas con discapacidad [actualizado 2015 Agosto; citado 2015 marzo]. Disponible en http://issuu.com/fercho77/docs/estadistica_02_de_agosto.

Corvera, J., F. González, 2000. Psicodinámia de la sordera. Gac. Med. Mex., 136(2), 139-152.

DesGeorges, J., 2003. Family perceptions of early hearing, detection, and intervention systems: Listening to and learning from families. Mental Retardation and Developmental Disabilities, Research Reviews, 9, 89-93.

Dominguez, A., 2009. Educación para la inclusión de alumnos sordos. Revista Latinoamericana de Educación Inclusiva, 3(1), 45-61.

Fahl, C., A. Gesueli, I. Rodrigues, 2013. A relacao sujeito, linguagem na construcao da identidade surda. Educ. Soc., 34(123), 509-527.

Fadda, S., 2011. Psycological aspects when counseling families who have children with cochlear implants. J. Matern. Fetal Neonatal Med., 24:S(1), 104-106.

Fuentes, M., 2000. Los numerales en la lengua de signos catalana. Revista de Lingüística, 12, 19-35.

Kobosko, J., 2011. Parenting a deaf child. How hearing parents cope with the stress of having deaf children. J. Hear. Sci., 1(3), 38-42.

Herrera, F., 2005. Habilidad lingüística y fracaso lector en los estudiantes sordos. Ensayos Estudios Pedagógicos, 1(2), 121-135.

Huerin, V., C. Duhalde, M.P. Vernengo, M.J. Esteve, J.A. Laplacette, C. Raznoszczyk, 2012. Regulación afectiva y simbolización. Estudio comparativo entre niños oyentes y no oyentes en edad preescolar. Anu. Investig., 19(2), 233-241.

Huiracocha, L., C. Almeida, K. Huiracocha, A. Arteaga, J. Arteaga, P. Barahona, J. Quezada, 2013. Explorando los sentimientos de los padres, la familia y la sociedad a las personas con Síndrome de Down: Estudio Observacional. Maskana, 4(2), 47-57.

Huiracocha, L., L. Brito, M.E. Pérez, R. Clavijo, S. Sempértegui, S. Blume, 2015. "Su guagua no escucha nada": Confronting the deafness of a child. Disabil. Soc., 30(4), 556-568.

Lederberg, A., T. Golbach, 2002. Parenting stress and social support in hearing mothers of deaf and hearing children: a longitudinal study. J. Deaf Stud. Deaf Educ., 7(4), 330-345. 
Langereis, M., A. Vermeulen, 2015. School performance and wellbeing of children with CI in different communicative- educational environments. Int. J. Pediatr. Otorhi., 79, 834-839.

Maddalena, V., F. O’Shea, M. Murphy, 2012. Palliative and end-on life care in Nwefoundland's deaf community. J. Palliat. Care, 28(2), 105-12.

Martin, D., Y. Bat-Chava, 2003. Negotiating deaf-hearing friendships: coping strategies of deaf boys and girls in mainstream schools. Child Care Health Dev., 29(6), 511-521.

Martinez, R., A. Landa, 2001. Niveles lectores de los niños sordos españoles. Revista de Psicología Universitas Tarraconensis, 23(1), 58-71.

Monsalve, A., 2002. La compleja apuesta en la educación de los niños sordos. Aula Abierta, 79, 59-67.

Moeller, M.P., G. Carr, L. Seaver, A. Stredler Brown, D. Holzinger, 2013. Best practices in family centered early intervention for children who are deaf or hard of hearing: an International Consensus Statement. J. Deaf Stud. Deaf Educ., 18(4), 429-445.

Muñoz-Baell, I, M.T. Ruiz-Cantero, C. Álvarez-Dardet, E. Ferreiro-Lago, E. Aroca-Fernández, 2011. Comunidades sordas: ¿pacientes o ciudadanas? Gac. Sanit., 25(1), 72-78.

Organización Mundial de la Salud, 2015. Sordera y pérdida de la audición, 2013 [actualizado 2015; citado marzo 2015]. Disponible en http://www.who.int/mediacentre/factsheets/fs300/es/.

Ortiz, M., 2006. El docente de niños sordos y el aprendizaje de la lectura y escritura. Un estudio de caso. Revista Latinoamericana de Lectura, 27(2), 14-21.

Plotkin, R., P. Brice, J. Reesman, 2014. It is not just stress: Parent personality in raising a deaf child. J. Deaf Stud. Deaf Educ., 19(3), 347-357.

Steinberg, A., S. Barnett, H. Meador, E. Wiggins, P. Zazove, 2006. Health Care System Accessibility: Experiences and perceptions of Deaf People. J. Gen. Intern Med., 21, 260-266.

Suárez, R., J. Rodríguez, V. Castro, 2006. La discapacidad auditiva: Impacto en la familia e importancia del apoyo social. Revista Qurriculum, 19, 221-232. 